\title{
ON INSTANT BLOW-UP FOR SEMILINEAR HEAT EQUATIONS WITH GROWING INITIAL DATA*
}

\author{
YOSHIKAZU GIGA ${ }^{\dagger}$ AND NORIAKI $\mathrm{UMEDA}^{\dagger}$ \\ Dedicated to Professor Neil S. Trudinger on the occasion of his 65th birthday
}

\begin{abstract}
For a semilinear heat equation admitting blow-up solutions a sufficient condition for nonexistence of local-in-time solutions are obtained. In particular, it is shown that if an initial data tends to infinity at space infinity then there is no local-in-time solution. As an application if the solution blows up at space infinity with least blow-up time, the solution cannot be extendable after blow-up time.
\end{abstract}

Key words. Instant blow-up, local-in-time solution, semilinear heat equation.

AMS subject classifications. 35K15, 35K55

1. Introduction and main theorems. We consider the initial value problem for a semilinear heat equation of the form

$$
\begin{cases}u_{t}=\Delta u+f(u), & x \in \mathbf{R}^{n}, t \in(0, T), \\ u(x, 0)=u_{0}(x), & x \in \mathbf{R}^{n} .\end{cases}
$$

Here we assume that the nonlinear term $f \in C^{1}(\mathbf{R})$ satisfies the following conditions:

$f$ is positive, nondecreasing and convex in $(0, \infty)$ and $\int_{1}^{\infty} \frac{d s}{f(s)}<\infty$.

The last condition guarantees that a positive constant solution blows up to infinity in finite time. A typical example of $f$ is $f(u)=e^{u},|u|^{p-1} u$ and $u_{+}\left(\log \left(u_{+}+1\right)\right)^{p}$ for $p>1$, where $u_{+}=\max \{u, 0\}$.

We are interested in the problem whether there is a local-in-time solution of (1) when an initial data $u_{0}$ grows at the space infinity, for example $\lim _{|x| \rightarrow \infty} u_{0}(x)=\infty$.

To be precise by a solution $u$ in $\mathbf{R}^{n} \times[0, T)$ of (1) we mean that $u \in C\left(\mathbf{R}^{n} \times\right.$ $[0, T)) \cap C^{2,1}\left(\mathbf{R}^{n} \times(0, T)\right)$ satisfies (1). For a given initial data $u_{0}$ let $T^{*}=T^{*}\left(u_{0}\right)$ be the maximal existence time of the solution. If $T^{*}=\infty$, the solution exists globally in time. If there are several solutions with the same initial data, we interpret that $T^{*}$ is the supremum of all existence times of these solutions. If $T^{*} \in(0, \infty)$, we often say that the solution blows up in finite time.

In this paper among other results we shall prove that $T^{*}=0$ when the initial data $u_{0}$ is growing at the space infinity. In other words there is even no local-in-time solution. We say this phenomenon $T^{*}=0$ an instant blow-up. We are able to prove that the instant blow-up occurs for more general initial data $u_{0}$.

Theorem 1. Assume (2) and that $u_{0} \in C\left(\mathbf{R}^{n}\right)$ is nonnegative. Assume that there are a sequence $\left\{x_{m}\right\} \subset \mathbf{R}^{n}$ with $\left|x_{m}\right| \rightarrow \infty$ as $m \rightarrow \infty$ and a number $r>0$ such that

$$
\lim _{m \rightarrow \infty} b_{m}=\infty, \text { with } b_{m}=\inf \left\{u_{0}(x):\left|x-x_{m}\right| \leq r\right\}
$$

\footnotetext{
*Received May 2, 2008; accepted for publication June 4, 2008.

${ }^{\dagger}$ Graduate School of Mathematical Sciences, University of Tokyo, 3-8-1, Komaba, Meguro-ku, Tokyo 153-8914, Japan (labgiga@ms.u-tokyo.ac.jp; umeda@ms.u-tokyo.ac.jp).
} 
Then $T^{*}=0$, i.e., the instant blow-up occurs provided that only nonnegative solutions are considered.

We may relax the assumption (3) so that $r=r_{m}$ depends on $m$ provided that

$$
\limsup _{m \rightarrow \infty} \frac{b_{m}}{r_{m}^{2} f\left(b_{m}\right)}<\epsilon
$$

with small $\epsilon>0$, say, $0<\epsilon<\epsilon_{0}$; the smallness constant $\epsilon_{0}$ depends only on the first eigenvalue of $-\Delta$ in a unit ball with the Dirichlet boundary condition so it depends only on the space dimension $n$. Note that this condition is automatically fulfilled if $r_{m}$ is independent of $m$ by our assumption (2), which in particular says that $f$ is superlinear. We may relax the assumption on nonnegativity of solution by lower boundedness of the solution in $\mathbf{R}^{n} \times\left[0, T^{\prime}\right]$ for any $T^{\prime}<T^{*}$ by a comparison principle provided that $f$ is $C^{1}$ in $\mathbf{R}$. The condition (3) is fulfilled when $\lim _{|x| \rightarrow \infty} u_{0}(x)=\infty$.

Our result applies to an extension problem of the solution after it blows up. For example it was proved in [5], [6] that the solution $w(x, t)$ blows up at $T^{*}=T^{*}(M)>0$ when $f(u)=|u|^{p-1} u$ or $e^{u}$ and the initial data $w_{0}$ satisfies $\lim _{|x| \rightarrow \infty} w_{0}(x)=M>0$. Moreover, blow-up occurs only at the space infinity. This implies that the profile function $w\left(x, T^{*}\right)$ at at $t=T^{*}$ is continuous (in fact $C^{2}$ ) and positive by a standard linear regularity theory [4] (which has an elliptic counterpart [7]). As in [5], [6] $\lim _{|x| \rightarrow \infty} w\left(x, T^{*}\right)=\infty$. If we interpret $w\left(x, T^{*}\right)$ as a new initial data, because of Theorem 1 it is impossible to extend the solution for $T>T^{*}$. We call this phenomenon non extendable blow-up. This is clearly related to the notion of 'complete blow-up' ([17], [13]), but it is not the same. If $w_{0}$ has a direction of mean convergence which is equivalent to say the solution has a least blow-up time, (i.e., the blow-up time $T^{*}$ agrees with the blow-up time of a spatially constant solution with an initial data $\sup w_{0}$ ), then the blow-up profile $w\left(x, T^{*}\right)$ has a blow-up direction (see [4]). As remarked in Appendix the $w\left(x, T^{*}\right)$ fulfills (3). Thus we always observe the non extendable blow-up.

For the space infinity blow-up the reader is refereed to papers [11], [8] [5], [6], [16] [18], [17], [15] and a review paper [4]. There is a nice book [13] for overview of blow up problems.

The reader may wonder whether there is a local-in-time solution with singularity. We say that $u \in C\left([0, T), L_{l o c}^{1}\left(\mathbf{R}^{n}\right)\right.$ is a strong $L_{l o c}^{1}$ solution of (1) with an initial data $u_{0} \in L_{l o c}^{1}$ if all terms in (1) are in $C\left((0, T) ; L_{l o c}^{1}\left(\mathbf{R}^{n}\right)\right)$ and satisfy (1) in distribution sense. Here $L_{l o c}^{1}$ is a Frechet space equipped with seminorm $|h|_{R}=\int_{B(0, R)}|h|(x) d x$, where $B(x, R)$ is an open ball of radius $R$ centered at $x$. We may replace a solution in Theorem 1 by a strong $L_{l o c}^{1}$ solution. The strong $L_{l o c}^{1}$ solution may have singularity. For example if $f(u)=u^{p}$ with $p>n /(n-2)$ for $n \geq 3$ then $u(x)=C_{p}|x|^{-2 /(p-1)}$ with $C_{p}=2((n-2) p-n) /(p-1)^{2}$ is a stationary strong $L_{l o c}^{1}$ solution of (1).

The proof of Theorem 1 depends on a classical Kaplan's argument [10] to show the existence of blow-up which uses principal eigenfunctions of the Laplace operator with the Dirichlet condition. We give another argument based on the energy principle developed by Ball [2] and Levine [12] together with a comparison argument. We need not assume positivity of the solution. We assume that a solution is bounded from below.

We assume that

$$
\text { there exists } \delta>0 \text { such that } s f(s) \geq(2+\delta) \int_{0}^{s} f(\xi) d \xi \text { for } s>0,
$$


and

$$
(s f(s))^{1 / 2} \text { is convex for } s>0, \int_{1}^{\infty} \frac{d \xi}{(\xi f(\xi))^{1 / 2}}<\infty .
$$

Let $u_{0}$ satisfy that there exists sequence $\left\{w_{0, m}\right\}_{m=1}^{\infty}$ with

$$
w_{0, m} \in L^{\infty} \cap H_{0}^{1}(B) \text { for } m=1,2, \ldots,
$$

such that for some $C \in \mathbf{R}$

$$
\begin{array}{r}
u_{0}\left(x+x_{m}\right) \geq w_{0, m}(x) \text { for } x \in B, \\
w_{0, m}(x)=C \text { for } x \in \partial B,
\end{array}
$$

and

$$
\lim _{m \rightarrow \infty}\left\{\int_{B}\left(\int_{C}^{w_{0, m}} f(s) d s-\frac{\left|\nabla w_{0, m}\right|^{2}}{2}\right) d x\right\}=+\infty,
$$

where $B=B(0, r)$ with some $r>0$.

We are now in position to state an instant blow-up result under a different setting.

THEOREM 2. Assume (2) and that $u_{0} \in C\left(\mathbf{R}^{n}\right)$ is nonnegative. Let $f$ satisfy (5) and (6). For $u_{0}$ assume that there exists a sequence $\left\{w_{0, m}\right\}_{m=1}^{\infty}$ with $w_{0, m} \in$ $L^{\infty} \cap H_{0}^{1}(B)$ satisfying (8), (9) and (10). Then $T^{*}\left(u_{0}\right)=0$ under the assumption that the solution is bounded from below in $\left[0, T^{\prime}\right]$ for any $T^{\prime}<T^{*}\left(u_{0}\right)$.

Note that the assumption (3) implies the existence of such $w_{m}$.

The authors were informed of a work Andreucci and Di Benedetto closely related to our present work. In [1] among other results a sufficient condition on initial data for nonexistence of a local-in-time nonnegative solution for $u_{t}=\Delta u^{m}+u^{p} /(1+|x|)^{\alpha}$ with $m \geq 1, p>1$ and $\alpha \in \mathbf{R}$. In the case of $m=1$ and $\alpha=0$ the condition reads

$$
\sup _{x \in \mathbf{R}^{n}} \int_{B(x, 1)} u_{0}(y) d y=\infty
$$

In [1] this is explicitly mentioned for $1<p<1+2 / n$. However, their proof is still valid for all $p>1$. By the way their main interest is the existence of solution; for example they proved the local existence when

$$
\sup _{x \in \mathbf{R}^{n}} \int_{B(x, 1)} u_{0}(y) d y<\infty
$$

for $1<p<1+2 / n$. Our Theorem 1 is included in their results for $f(u)=u^{p}$. However, the condition (4) is not included in their result even for $f(u)=u^{p}$ for $p>1+2 / n$. In fact, if $u_{0} \geq b_{m}$ on $B\left(x_{m}, r_{m}\right)$, then $\lim _{m \rightarrow \infty} b_{m} r_{m}^{n}=\infty$ is a sufficient condition for (11). Our condition (4) with $f(u)=u^{p}$ reads $\lim _{m \rightarrow \infty} r_{m}^{2} b_{m}^{p-1}=\infty$. This shows that our condition for $p>1+2 / n$ is weaker than their condition.

In [1] they also prove the local existence for $p \geq 1+2 / n$ when $u_{0}$ fulfills

$$
\sup _{x \in \mathbf{R}^{n}} \int_{B(x, 1)} u_{0}^{q}(y) d y<\infty
$$


for some $q>n(p-1) / 2$. Our result suggests the nonexistence when $u_{0}$ satisfies

$$
\sup _{x \in \mathbf{R}^{n}} \int_{B(x, 1)} u_{0}^{n(p-1) / 2}(y) d y \text { is sufficiently large. }
$$

The rest of the paper is organized as follows. In Section 2 we show Theorem 1 by using the argument in [10]. Theorem 2 is proved by the method of [2] and [12] in Section 3. In Appendix we show that if the solution has a blow-up direction, the blow-up profile satisfies (3).

2. Eigenfunction method. We begin by recalling a comparison result.

Lemma 2.1 (Comparison). Assume (2) and that $f \in C^{1}(\mathbf{R})$. Let $u(x, t)$ and $v(x, t)$ be solutions of (1) with initial data $u_{0}$ and $v_{0}$ which are continuous in $\mathbf{R}^{n}$. Assume that $v$ is bounded in $\mathbf{R}^{n} \times\left[0, T^{\prime}\right)$ for any $T^{\prime}<T$. Assume that $u$ is bounded from below in $\left\{\mathbf{R}^{n} \times\left[0, T^{\prime}\right)\right\}$ for any $T^{\prime}<T$. If $u_{0} \geq v_{0}$ in $\mathbf{R}^{n}$, then $u \geq v$ in $\mathbf{R}^{n} \times(0, T)$.

Proof. The proof is based on an maximum principle for a parabolic equation and is standard (see [9], [3] and [14]). We give it for completeness.

We may assume that $v$ is bounded and continuous in $\mathbf{R}^{n} \times[0, T]$ by taking $T$ smaller. We may assume that $u$ is continuous in $\mathbf{R}^{n} \times[0, T]$. Suppose that the conclusion were false. Then there would exist a point $(\hat{x}, \hat{y}) \in \mathbf{R}^{n} \times(0, T)$ such that $w(\hat{x}, \hat{t})>0$ for $w=v-u$. We set

$$
Q=\left\{(x, t) \in \mathbf{R}^{n} \times(0, T): w(x, t)>0\right\} .
$$

Since $u<v$ in $Q$ and $v$ is bounded, we see that $u$ is bounded in $Q$ since we have assumed that $u$ is bounded from below. By our assumption $Q$ is a bounded open set in $\mathbf{R}^{n} \times(0, T)$. Subtracting (1) for $u$ from (1) for $v$ we obtain

$$
w_{t}=\Delta w+b(x, t) w \quad \text { in } Q
$$

with $b(x, t)=\int_{0}^{1} f^{\prime}(u(x, t)+\theta(v(x, t)-u(x, t))) d \theta$. Since $u$ is bounded in $Q$, we have $M_{v}=\sup _{Q} b<\infty$. Thus $w$ solves

$$
\begin{cases}w_{t} \leq \Delta w+M_{v} w, & (x, t) \in Q \\ w=0, & (x, t) \in \partial Q .\end{cases}
$$

We set $W(x, t)=e^{-\left(M_{v}+1\right) t} w(x, t)$ to set

$$
\begin{cases}W_{t} \leq \Delta W-W, & (x, t) \in Q, \\ W=0, & (x, t) \in \partial Q .\end{cases}
$$

(If $Q$ is bounded, we immediately conclude that $W$ cannot take a possible maximum in $Q$. However, $Q$ may be unbounded so we modify $W$.)

We set $W_{\epsilon}=W-\epsilon\left(|x|^{2}+A t\right)$ with $A=2 n+1$ and small $\epsilon>0$ to be determined later. It follow that

$$
\left(\partial_{t}-\Delta+1\right) W_{\epsilon}<0, \quad(x, t) \in Q .
$$

For $(\tilde{x}, \tilde{t}) \in Q$ there exist $\alpha>0$ such that $W(\tilde{x}, \tilde{t})>\alpha$. Let $\epsilon>0$ small enough. Then we see

$$
W_{\epsilon}(\tilde{x}, \tilde{t})=W-\epsilon\left(|\tilde{x}|^{2}+A \tilde{t}\right) \geq \frac{\alpha}{2}>0
$$


as well as $w=v-u$. We now put $R=\epsilon^{-1 / 2}(\sup W)^{1 / 2}$. It is easily seen that $W_{\epsilon}(x, t)<0$ for $|x|>R$. Since $W_{\epsilon}$ is continuous in $Q$, There exists $(\hat{x}, \hat{t}) \in \bar{B}_{R} \times$ $[0, T) \cap Q$ such that

$$
W_{\epsilon}(\hat{x}, \hat{t})=\sup \left\{W_{\epsilon}(x, t) \in \bar{B}_{R} \times[0, T] \cap Q\right\} .
$$

It follows that

$$
\left(W_{\epsilon}\right)_{t}(\hat{x}, \hat{t}) \geq 0, \Delta W_{\epsilon}(\hat{x}, \hat{t}) \leq 0, \text { and } W_{\epsilon}(\hat{x}, \hat{t}) \geq 0
$$

Thus we have

$$
\left(\partial_{t}-\Delta+1\right) W_{\epsilon}(\hat{x}, \hat{t}) \geq 0
$$

and we have a contradiction to (15). We thereby get

$$
w(x, t) \leq 0
$$

and $u(x, t) \geq v(x, t)$.

Proof of Theorem 1. Let $\left\{b_{m}\right\}_{m=1}^{\infty}$ and $\left\{x_{m}\right\}_{m=1}^{\infty}$ be as in Theorem 1 with $r=r_{m}$ satisfying (4). Set $\lambda_{m}>0$ denote the principal eigenvalue of $-\Delta$ with Dirichlet problem in $B_{r_{m}}(0)$, and let $\phi_{m}(x) \geq 0$ denote the corresponding positive eigenfunction normalized by $\int_{B_{r_{m}}(0)} \phi_{m}(x) d x=1$. By scaling it is easy to observe that

$$
\lambda_{m}=\frac{c}{r_{m}^{2}}
$$

with some $c>0$. Define

$$
G_{m}(t)=\int_{B\left(x_{m}, r_{m}\right)} u(x, t) \phi_{m}\left(x-x_{m}\right) d x .
$$

Let $n_{m}(x)$ denote the outward unit normal to $B\left(0, r_{m}\right)$ at $x \in \partial B\left(0, r_{m}\right)$. Integrating by parts, by the fact that $\phi_{m}=0$ and $\partial \phi_{m} / \partial n_{m} \leq 0$ on $\partial B\left(0, r_{m}\right)$ with the unit normal vector $n_{m}$, and applying Green's formula and Jensen's inequality, we obtain

$$
\begin{aligned}
G_{m}^{\prime}(t) & =\int_{B\left(x_{m}, r_{m}\right)} u_{t}(x, t) \phi_{m}\left(x-x_{m}\right) d x \\
& \geq \int_{B\left(x_{m}, r_{m}\right)}\left\{(\Delta u(x, t)+f(u(x, t))\} \phi_{m}\left(x-x_{m}\right) d x\right. \\
& \geq-\lambda_{m} G_{m}(t)+f\left(G_{m}(t)\right) .
\end{aligned}
$$

Thus, we obtain

$$
G_{m}^{\prime}(t) \geq-\lambda_{m} G_{m}(t)+f\left(G_{m}(t)\right)
$$

Let us consider the system of ordinary differential equations

$$
\left\{\begin{array}{l}
g_{m}^{\prime}(t)=-\lambda_{m} g_{m}(t)+f\left(g_{m}(t)\right) \\
g_{m}(0)=G_{m}(0) \geq b_{m}
\end{array}\right.
$$


Define $T_{g_{m}}=\sup \left\{t \geq 0: g_{m}(t)<\infty\right\}$ and $T_{G_{m}}=\sup \left\{t \geq 0: G_{m}(t)<\infty\right\}$. Since $G_{m} \geq g_{m}$, we obtain $T_{g_{m}} \geq T_{G_{m}}$. If $r_{m}$ is a constant so that $\lambda_{m}=\lambda$ is depend of $m$, then

$$
T_{g_{m}} \leq \int_{b_{m}}^{\infty} \frac{d \xi}{-\lambda \xi+f(\xi)} \rightarrow 0 \text { as } m \rightarrow \infty .
$$

This implies that $T_{G_{m}} \rightarrow 0$ as $m \rightarrow \infty$. In particular for sufficiently large $m, T_{G_{m}}<T$. This is a contradiction since $u$ is continuous in $\mathbf{R}^{n} \times[0, T)$.

We shall discuss the case that $r_{m} \rightarrow 0$ as $m \rightarrow \infty$ satisfying (4). Consider the solutions of (1) with the initial data $b_{m}$. The maximal existence time of the solution denoted by $T^{*}\left(b_{m}\right)$ is estimated as

$$
T^{*}\left(b_{m}\right)=\int_{b_{m}}^{\infty} \frac{d \xi}{f(\xi)} .
$$

Note that $\lim _{m \rightarrow \infty} T^{*}\left(b_{m}\right)=0$. Consider the formula;

$$
\frac{T^{*}\left(b_{m}\right)}{T_{g_{m}}}=\frac{\int_{b_{m}}^{\infty} d \xi / f(\xi)}{\int_{b_{m}}^{\infty} d \xi /\left(-\lambda_{m} \xi+f(\xi)\right)} .
$$

From (4) we may assume that there exist $m_{0} \geq 0$ such that

$$
\frac{b_{m}}{r_{m}^{2} f\left(b_{m}\right)}<\epsilon
$$

for $m \geq m_{0}$. From (17) we see that

$$
\lambda_{m} b_{m}<c \epsilon f\left(b_{m}\right) .
$$

Since $f$ satisfies (2), we get

$$
\lambda_{m} \xi<c \epsilon f(\xi)
$$

for $\xi \geq b_{m}$. Substituting (21) for (20), we have

$$
\frac{T^{*}\left(b_{m}\right)}{T_{g_{m}}}>1-c \epsilon
$$

for $m \geq m_{0}$. Thus we obtain

$$
\lim _{m \rightarrow \infty} \frac{T^{*}\left(b_{m}\right)}{T_{g_{m}}}>1-c \epsilon>0 .
$$

Noting that $\lim _{m \rightarrow \infty} T^{*}\left(b_{m}\right)=0$, we see that $\lim _{m \rightarrow \infty} T_{g_{m}}=0$. Again we get $T_{G_{m}} \rightarrow 0$ as $m \rightarrow \infty$ which is a contradiction.

REMARK 2.2. So far we did not use Lemma 2.1. Even if we consider the sign changing solution for the nonnegative initial data, by comparison (Lemma 2.1) it must be nonnegative provided that it is bounded from below in $\mathbf{R}^{n} \times\left[0, T^{\prime}\right]$ for any $T^{\prime}<T$. 
3. Energy method. In this section, we prove Theorem 2. We may assume that $C=0$ in (8), (9) and (10).

For $m=1,2, \ldots$, we consider a problem:

$$
\begin{cases}\left(w_{m}\right)_{t}=\Delta w_{m}+f\left(w_{m}\right), & x \in B, t>0, \\ w_{m}(x, 0)=w_{0, m}(x), & x \in B \\ w_{m}=0 & x \in \partial B\end{cases}
$$

where $w_{0, m} \in L^{\infty} \cap H_{0}^{1}(B)$ satisfies (8), (9) and (10), and $B=B(0,1)$

By comparison in $B$ we have

$$
u\left(x+x_{m}, t\right) \geq w_{m}(x, t) \quad \text { in } B
$$

for $m=1,2, \ldots$. Put

$$
\phi_{m}(t)=\int_{B} w_{m}^{2}(x, t) d x
$$

and

$$
E_{m}(t)=\int_{B}\left(\frac{|\nabla w|^{2}}{2}-\int_{0}^{w} f(\xi) d \xi\right) d x .
$$

The ideas of the proof of next two lemmas are standard and go back to [2] and [12]. We give it for completeness.

Lemma 3.1 (Monotonicity of energy). Let $E_{m}(t)$ be as in (24). Then

$$
E_{m}^{\prime}(t) \leq 0 \text {. }
$$

Proof. From the proof of [13, Lemma 17.5] and the fact that

$$
\frac{d}{d t}\left(\int_{0}^{w} f(\xi) d \xi\right)=f(w) w_{t}
$$

we get

$$
E_{m}^{\prime}(t)=-\int_{B} w_{t}^{2}(x, t) d x \leq 0
$$

Lemma 3.2 (Differential inequality For $L^{2}$ NORM). Define $\phi_{m}$ and $E_{m}$ in (23) and (24). Then

$$
\phi_{m}^{\prime}(t) \geq-2 E_{m}(0)+c g\left(\phi_{m}(t)\right)
$$

with $g(\xi)=(\xi f(\xi))^{1 / 2}$ and $c=c(\delta)$, where $\delta$ is defined in (5).

Proof. Differentiating (23) with respect to $t$ and multiplying $1 / 2$, we have

$$
\begin{aligned}
\frac{1}{2} \phi_{m}^{\prime}(t) & =\int_{B} w w_{t} d x \\
& =\int_{B} w(\Delta w+f(w)) d x \\
& =\int_{B}\left(-|\nabla w|^{2}+w f(w)\right) d x
\end{aligned}
$$


From (5) we obtain

$$
\begin{aligned}
\frac{1}{2} \phi_{m}^{\prime}(t) & \geq \int_{B}\left(-|\nabla w|^{2}+\delta w f(w)+2 \int_{0}^{w} f(\xi) d \xi\right) d x \\
& =-2 E_{m}(t)+\int_{B} \delta w f(w) d x
\end{aligned}
$$

From Lemma 3.1 and the fact that $g(\xi)=(\xi f(\xi))^{1 / 2}$ is convex, we have

$$
\begin{aligned}
\frac{1}{2} \phi_{m}^{\prime}(t) & \geq-2 E(0)+c g\left(\int_{B} w^{2} d x\right) \\
& =-2 E(0)+c g\left(\phi_{m}\right)
\end{aligned}
$$

by Jensen's inequality, where $c=\delta|B|$.

Lemma 3.3. Assume that $g \in C[0, \infty)$ is positive, nondecreasing and convex in $[0, \infty)$. Assume that $\int_{1}^{\infty} d \xi / g(\xi) \leq C<\infty$. Then there exists a sequence $\left\{\eta_{m}\right\}_{m=1}^{\infty}$ such that $\lim _{m \rightarrow \infty} \eta_{m}=\infty$ and

$$
\lim _{m \rightarrow \infty} \int_{1}^{\infty} \frac{d \xi}{\eta_{m}+g(\xi)}=0
$$

Proof. Assume that

$$
\lim _{m \rightarrow \infty} \int_{1}^{\infty} \frac{d \xi}{\eta_{m}+g(\xi)} \geq \epsilon>0
$$

Then for any $M>1$

$$
\lim _{m \rightarrow \infty} \int_{M}^{\infty} \frac{d \xi}{\eta_{m}+g(\xi)} \geq \frac{\epsilon}{2} \quad \text { or } \quad \lim _{m \rightarrow \infty} \int_{1}^{M} \frac{d \xi}{\eta_{m}+g(\xi)} \geq \frac{\epsilon}{2} .
$$

However, for any $\epsilon>0$ we can take $M>1$ large enough such that

$$
\lim _{m \rightarrow \infty} \int_{M}^{\infty} \frac{d \xi}{g(\xi)}<\frac{\epsilon}{2}
$$

Thus we obtain

$$
\lim _{m \rightarrow \infty} \int_{M}^{\infty} \frac{d \xi}{\eta_{m}+g(\xi)} \leq \lim _{m \rightarrow \infty} \int_{M}^{\infty} \frac{d \xi}{g(\xi)}<\frac{\epsilon}{2}
$$

On the other hand, for any $M>1$

$$
\begin{aligned}
\int_{1}^{M} \frac{d \xi}{\eta_{m}+g(\xi)} & =\int_{1}^{M} \frac{g(\xi)}{\eta_{m}+g(\xi)} \cdot \frac{d \xi}{g(\xi)} \leq \frac{g(M)}{\eta_{m}+g(M)} \int_{1}^{M} \frac{d \xi}{g(\xi)} \\
& \leq \frac{C g(M)}{\eta_{m}+g(M)} \rightarrow 0 \text { as } m \rightarrow 0 .
\end{aligned}
$$

Thus we have

$$
\lim _{m \rightarrow \infty} \int_{1}^{M} \frac{d \xi}{\eta_{m}+g(\xi)}<\frac{\epsilon}{2}
$$


for any $M>1$. We thereby have a contradiction. Thus we obtain

$$
\lim _{m \rightarrow \infty} \int_{1}^{\infty} \frac{d \xi}{\eta_{m}+g(\xi)}=0
$$

Proof of Theorem 2. By Lemma 2.1 we may assume that $u$ is nonnegative. From Lemma 3.2 we have

$$
\phi_{m}^{\prime}(t) \geq-2 E_{m}(0)+c g\left(\phi_{m}(t)\right) .
$$

If $E_{m}(0)<0$ and $\phi_{m}(0)>0$, then there exist a constant $T_{m}>0$ such that $\lim _{t \rightarrow T_{m}} \phi_{m}(t)=\infty$, and

$$
T_{m} \leq \int_{\phi_{m}(0)}^{\infty} \frac{d \xi}{-2 E_{m}(0)+c g(\xi)} .
$$

From (10) we see that

$$
\lim _{m \rightarrow \infty} E_{m}(0)=-\infty
$$

Thus from Lemma 3.3 we obtain

$$
\lim _{m \rightarrow \infty} T_{m}=0 .
$$

Since $u \geq 0$, by a comparison in $B$ we have $T^{*} \leq T_{m}$. This implies $T^{*}\left(u_{0}\right)=0$.

4. Appendix. In this section we shall show that if $u_{0}$ is a profile at blow-up having a blow-up direction in the sense of [6] and continuous, then (3) is fulfilled. We consider the solution $w$ of the initial value problem (1) with an initial data $w_{0}$ having a direction $\psi \in S^{n-1}$ of means convergence in [4]. One of equivalent definitions reads: there exists a positive constant $M$ such that $0 \leq w_{0} \leq M$ and

$$
\inf _{x \in B\left(x_{m}, r_{m}\right)}\left(w_{0}(x)-M_{m}\right) \geq 0
$$

with sequences $\left\{r_{m}\right\}_{m=1}^{\infty} \subset(0, \infty),\left\{x_{m}\right\}_{m=1}^{\infty} \subset \mathbf{R}^{n}$, and $\left\{M_{m}\right\}_{m=1}^{\infty}$ satisfying $r_{m} \rightarrow$ $\infty, M_{m} \rightarrow M$ as $m \rightarrow \infty$. It turns out that this condition is equivalent to say that the solution has a least blow-up time (see [16], [4]).

From [16, Theorem 1.5] and [4, Theorem 3.2] the solution $w$ satisfies that for each $R>0$

$$
\lim _{m \rightarrow \infty} \sup _{x \in B\left(x_{m}, R\right)}(v(t)-w(x, t))=0,
$$

where $v$ is a solution of (1) with an initial data $M$, and the solution $w$ has a blowup direction at $t=T$. In other words there exist a direction $\psi \in S^{n-1}$, sequences $\left\{x_{m}\right\}_{m=1}^{\infty}$ and $\left\{t_{m}\right\}_{m=1}^{\infty}$ such that $x_{m} /\left|x_{m}\right| \rightarrow \psi$ and $w\left(x_{m}, t_{m}\right) \rightarrow \infty$ as $m \rightarrow \infty$.

If we let $u_{0}(x)=w\left(x, T^{*}\right)=\lim _{t \rightarrow T^{*}} w(x, t)$ with $T^{*}=T^{*}(M)$, then $u_{0}$ has a blow-up direction.

Lemma 4.1. Assume that $w_{0} \in C\left(\mathbf{R}^{n}\right)$ has a direction of mean convergence. Let $w$ be the solution of (1) with an initial data $w_{0}$. Then the blow-up profile $u_{0}(x)=$ $w\left(x, T^{*}\right)$ fulfills the assumption (3) of Theorem 1. 
Proof. From (25) we see that there exists a sequence $\left\{\left(x_{m}, t_{m}\right)\right\}_{m=1}^{\infty}$ satisfying $t_{m} \rightarrow T^{*}$ as $m \rightarrow \infty$ such that

$$
\lim _{m \rightarrow \infty}\left\{\sup _{x \in B\left(x_{m}, R\right)}\left(v\left(t_{m}\right)-u\left(x, t_{m}\right)\right)\right\}=0 .
$$

Thus, since $\lim _{m \rightarrow \infty} v\left(t_{m}\right)=\infty$, we have

$$
\lim _{m \rightarrow \infty}\left\{\inf _{x \in B\left(x_{m}, R\right)} u\left(x, t_{m}\right)\right\}=\infty
$$

for any $x \in B(0, R)$. We set $b_{m}=\inf _{x \in B\left(x_{m}, R\right)} u\left(x, t_{m}\right)$. Then $u_{0}(x)=w\left(x, T^{*}\right)$ satisfies (3).

Acknowledgement. The authors are grateful to Professor Ryuichi Suzuki for pointing out that our result is closely related to some of results in [1]. The work of the first author is partly supported by the Grant-in-Aid for Scientific Research, No.18204011, the Japan Society for the Promotion of Science (JSPS). Much of the work of the second author was done while he visited the Hokkaido University during 2003-2005 and the University of Tokyo during 2005-2008 as a postdoctoral fellow. Its hospitality is gratefully acknowledged as well as support from formation of COE "Mathematics of Nonlinear Structures via Singularities" (Hokkaido University) and COE "New Mathematical Development Center to Support Scientific Technology" (University of Tokyo), supported by JSPS.

\section{REFERENCES}

[1] D. Andreucci and E. Di Benedetto, On the Cauchy problem and initial trances for a class of evolution equations with strongly nonlinear sources, Ann. Scuola Norm. Sup. Pisa, 18 (1991), pp. 363-441.

[2] J. M. BALL, Remarks on blow-up and nonexistence theorems for nonlinear evolution equations, Quart. J. Math. Oxford Ser. (2), 28 (1977), pp. 473-486.

[3] M.-H. Giga And Y. Giga, Nonlinear Partial Differential Equations (in Japanese), Kyoritsu Shuppan ,Tokyo, Japan, 1999.

[4] Y. Giga, Y. Seki ANd N. Umeda, Blow-up at space infinity for nonlinear heat equation, Recent Advances in Nonlinear Analysis (eds. M. Chipot et. al.), pp. 77-94, World scientific, New Jersey, 2007.

[5] Y. Giga And N. Umeda, On blow-up at space infinity for semilinear heat equations, J. Math. Anal. Appl., 316 (2006), pp. 538-555.

[6] Y. Giga And N. Umeda, Blow-up directions at space infinity for solutions of semilinear heat equations, Bol. Soc. Parana. Mat. (3), 23 (2005), pp. 9-28.

[7] D. Gilbarg and N. S. Trudinger, Elliptic Partial Differential Equations of Second Order, Springer, 1977.

[8] A. L. Gladkov, Behavior of solutions of semilinear parabolic equations as $x \rightarrow \infty$, Mathematical Notes, 51 (1992), pp. 124-128.

[9] A. M. Il'in, A. S. Kalashnikov and O. A. Oleinik, Second-order linear equations of parabolic type, J. Math. Sci., 108 (2002), pp. 435-542. See also: Linear equations of the second order of parabolic type, Russ. Math. Surv., 17 (1962) (3), pp. 1-143. Both papers are translations of the original Russian version published in 'Uspekhi Mat. Nauk, 17 (1962), no. 3 (105), pp. $3-146$ '.

[10] S. Kaplan, On the growth of solutions of quasi-linear parabolic equations, Comm. Pure Appl. Math., 16 (1963), pp. 305-330.

[11] A. A. LACEY, The form of blow-up for nonlinear parabolic equations, Proc. Roy. Soc. Edinburgh Sect. A, 98 (1984), pp. 183-202.

[12] H. A. Levine, Some nonexistence and instability theorems for solutions of formally parabolic equations of the form $P u_{t}=-A u+\mathcal{F}(u)$, Arch. Rational Mech. Anal., 51 (1973), pp. 371386. 
[13] P. Quittner And P. Souplet, Superlinear parabolic problems, Blow-up, global existence and setady states, Birkhause, Besel-Boston-Berlin, 2007.

[14] O. SAwAdA AND Y. TANIUChI, A remark on $L^{1}$ solutions to the 2-D Navier-Stokes equations, J. Math. Fluid Mech., 9 (2007), pp. 533-542.

[15] Y. SEKI, On directional blow-up for quasilinear parabolic equations with fast diffusion, J. Math. Anal. Appl., 338 (2008), pp. 572-587.

[16] Y. SeKi, R. Suzuki and N. UmedA, Blow-up directions for quasilinear parabolic equations, Proc. Roy. Soc. Edinburgh Sect. A Math., 138A (2008), pp. 379-405.

[17] M. Shimojō, The global profile of blow-up at space infinity in semilinear heat equations, to appear in J. Math. Kyoto Univ.

[18] M. ShimoJō, On blow-up phenomenon at space infinity and its locality for semilinear heat equations (in Japanese), Master's Thesis, The University of Tokyo (2005). 
